\title{
FOUR-DIMENSIONAL LANGEVIN DYNAMICS OF HEAVY-ION-INDUCED FISSION
}

\author{
Nadtochy P.N., Fedorov S.V., Adeev G.D. \\ Omsk State University, Russia \\ E-mail: fsmail1987@gmail.com
}

A four-dimensional dynamical model based on Langevin equations was developed and applied to study fission characteristics in a wide range of a fissility parameter and excitation energy [1].The evolution of three collective shape coordinates and $K$-coordinate (spin projection onto symmetric axis of fission nucleus) [2], were considered from the ground state deformation to the scission of compound nucleus into fragments. A modified one-body mechanism of nuclear dissipation with a reduction coefficient $k_{s}$ of the contribution from a "wall" formula has been used in the study for modeling nuclear viscosity. The modeling of four collective coordinates allows calculating a wide set of experimental observables in fusion-fission reactions induced by heavy-ions [3]. The inclusion of $K$-coordinate in the dynamical consideration and use of the "chaos-weighted wall formula" with a deformation-depended scaling factor $k_{s}\left(q_{1}\right)$ lead to fairly good reproduction of variances of fission fragment mass distribution and prescission neutron multiplicity for a number of fissioning compound nuclei in a wide fissility range [4]. The four-dimensional dynamical calculations describe better experimental prescission neutron multiplicity and variances of fission fragment mass distribution for heaviest nuclei with respect to a three-dimensional dynamical model, where $K$-coordinate assumed to be equal to zero [5]. The estimate of a dissipation coefficient for the orientation degree of freedom $\gamma_{K} \simeq 0.77(\mathrm{MeV} \mathrm{zs})^{1 / 2}$ is good for heavy nuclei and a larger value of $\gamma_{K} \simeq 0.2(\mathrm{MeV} \mathrm{zs})^{1 / 2}$ is needed for the nuclei with mass $A_{C N} \simeq 200$.

1. P.N.Nadtochy et al.// Phys. Rev. C. 2012. V.85. 064619.

2. J.P.Lestone, S.G.McCalla// Phys. Rev. C. 2009. V.79. 044611.

3. K.Mazurek et al. // Phys. Rev. C. 2013. V.88. 054614.

4. P.N.Nadtochy et al.// Phys. Rev. C. 2014. V.89. 014616.

5. P.N.Nadtochy, G.D.Adeev, A.V.Karpov// Phys. Rev. C. 2002. V.65. 064615. 